\title{
NEW SEMI-ANALYTIC THEORY OF THE FOUR OUTER PLANETS
}

\author{
J.L. SIMON \\ Bureau des longitudes \\ 77, avenue Denfert-Rochereau \\ 75014, Paris FRANCE
}

\section{Introduction}

A planetary theory of the planets Jupiter, Saturn, Uranus and Neptune is presented here. It is a classical planetary theory where the perturbations are computed in the form of Poisson series of only one angular variable. It is built with modern values of the planetary masses and fitted to the numerical integration DE245 of the Jet Propulsion Laboratory (Standish, 1994). Its validity time span is of several thousand of years.

\section{Form of the solution}

\subsection{NOTATIONS}

The classical elliptic variables are used: $a, \lambda, e, \varpi, \gamma=\sin \frac{i}{2}, \Omega, k=e \cos \varpi$, $h=e \sin \varpi, q=\gamma \cos \Omega, p=\gamma \sin \Omega$. The mean mean longitude $\bar{\lambda}$ is given by: $\bar{\lambda}=\lambda^{0}+N t$ where $\lambda^{0}$ is the integration constant for the mean longitude $\lambda, N$ is the mean mean motion and $t$ is the time. The planets Jupiter, Saturn, Uranus and Neptune are numbered from 5 to 8.

\subsection{THE REPRESENTATION}

In a classical planetary theory the variables $x$ are developed under the form of Poisson series:

$$
x=x_{0}+x_{1} t+\ldots+x_{j} t^{j}+S_{0}+t S_{1}+\ldots+t^{j} S_{j}
$$

where: $x_{0}$ is the integration constant for $x, t$ is the time, $x_{q}$ are numerical coefficients, $S_{q}$ are Fourier series in the mean mean longitudes. 
Here, the solutions are also Poisson series of the form (1), but $S_{q}$ are Fourier series in only one argument $\mu$ :

$$
S_{q}=\sum_{r}\left\{A_{r} \cos r \mu+B_{r} \sin r \mu\right\}
$$

with: $\mu=\left(N_{5}-N_{6}\right) t / 880=0.3595 t$, where $t$ is measured in thousands of years from J2000. The period of $\mu$ is 17485 years.

$\mu$ is related to the mean mean longitudes $\bar{\lambda}_{i}$ by: $\bar{\lambda}_{i}=q_{i} \mu+\varepsilon_{i} t$, where $q_{i}$ are integers and where $\varepsilon_{i}$ are small quantities compared with $N_{i}$.

This representation was choosen because perturbations are more convergent under this form than under the classical form of Poisson series of the mean mean longitudes, for Jupiter and Saturn (Simon et al., 1992). For the other planets the convergence of perturbations is the same than under the classical form.

\section{Construction of the solution}

\subsection{THE METHOD}

The solution is computed by the iterative method of (Simon and Joutel, 1988). The equations are Lagrange equations under the form: $\frac{d x}{d t}=F(\mu, t)$ $F\left(\mu, t_{i}\right)$ are computed, by harmonical analysis, for 11 values of the time $t_{0},(\mathrm{~J} 2000), t_{1}, \ldots, t_{10}$. After interpolation and integration the solution has the form (1) with $j=10$. For each iteration, the truncation error is $0^{\prime \prime} .0001$ over $-1000,+1000(0=\mathrm{J} 2000)$ or $0^{\prime \prime} .01$ over $-6000,+6000$.

\subsection{PERTURBATIONS BY THE INNER PLANETS}

Perturbations by the inner planets developed up to the third order of the masses are issued from VSOP82 (Bretagnon, 1982). They are transformed in series in $\mu$ and added to the solution. Then, they are introduced in the iterations. So, are computed the perturbations by the inner planets to the order 4 of the masses and a part of the perturbations of superior orders.

\subsection{DETERMINATION OF THE INTEGRATION CONSTANTS}

This theory is built using the IERS Standards 1992 masses of the outer planets (McCarthy, 1992) and it is fitted to DE245. Note that the masses of the outer planets used in DE245 are the masses of IERS 1992, except a very small difference for the mass of Saturn. 


\section{Results}

\subsection{DIFFERENCES WITH DE245}

Table 1 gives the differences, after fit, between our theory and DE245 for the heliocentric variables, longitude, latitude and radius vector, over 18912000. These differences are small: some $0^{\prime \prime} .0001$ for the longitudes and the latitudes and some $10^{-8}$ au for the radius vector. Note that a large part of these differences is probably due to the truncation error. So, a few iterations with a truncation error of $0^{\prime \prime} .00001$ over $-1000,+1000$ are intended.

TABLE 1. Differences between our theory and DE245 for the heliocentric coordinates, over 1891-2000.

\begin{tabular}{lccc}
\hline & $\begin{array}{c}\text { Longitude } \\
\left(0^{\prime \prime} .001\right)\end{array}$ & $\begin{array}{c}\text { Latitude } \\
\left(0^{\prime \prime} .001\right)\end{array}$ & $\begin{array}{c}\text { Radius Vector } \\
\left(10^{-8} \mathrm{au}\right)\end{array}$ \\
\hline Jupiter & 5 & 2 & 7 \\
Saturn & 8 & 3 & 19 \\
Uranus & 8 & 5 & 50 \\
Neptune & 4 & 2 & 59 \\
\hline
\end{tabular}

\subsection{PRECISION OF THE THEORY}

The precision of our theory is computed by comparison to internal numerical integration of the eight major planets run over J2000-6000, J2000+6000. Figure 1 shows the differences between the theory and the numerical integration for the mean longitudes. For Jupiter, Uranus and Neptune the differences are smaller than $0^{\prime \prime} .01$ over $-1000,+1000$ and smaller than $0^{\prime \prime} .8$ over $-6000,+6000$. For Saturn, the precision is about $0^{\prime \prime} .02$ over $-1000,+1000$ and about $2^{\prime \prime}$ over $-6000,+6000$. For the other elliptic variables, the differences theory-numerical integration are of some $0^{\prime \prime} .001$ over $-1000,+1000$ and smaller than $0^{\prime \prime} .5$ over $-6000,+6000$.

The gain in precision, in connection with the best theories actually available, as VSOP87 (Bretagnon and Francou, 1987) and JASON84 (Simon and Bretagnon, 1984), is about 10.

\section{Conclusion}

We are going to obtain a semi-analytic theory of the outer planets built with modern values of the planetary masses, fitted to DE245 and showing real progress compared to the theories actually available.

Besides, due to the best convergence of the mutual perturbations JupiterSaturn, compact solutions, useful for historians, could be extracted from our theory. 


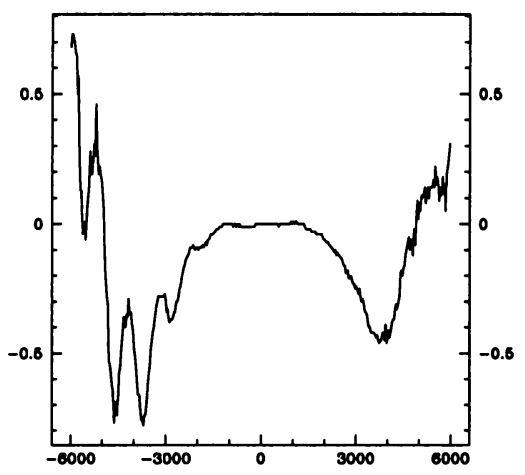

Mean longitude of Jupiter, unit: 1"

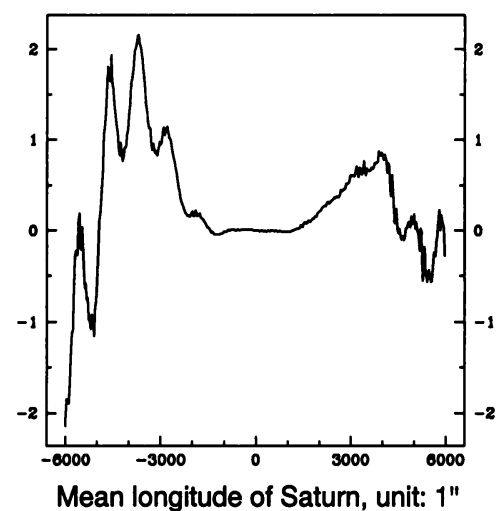

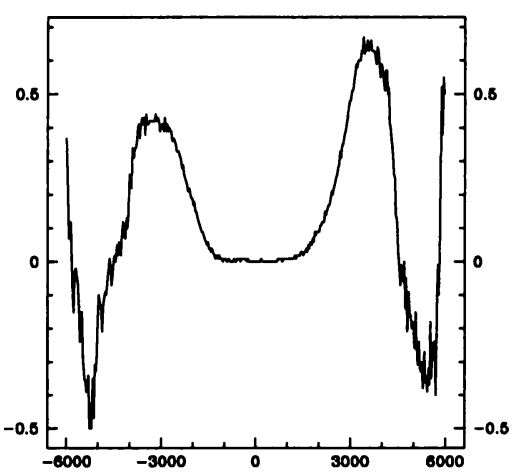

Mean longitude of Uranus, unit: 1"

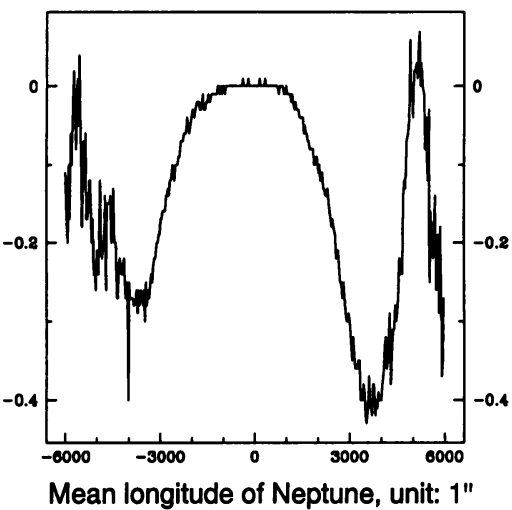

Figure 1. Mean longitudes of the outer planets. Comparison between our theory and an internal numerical integration of the eight major planete over $-6000,+6000$. 0 is J2000.

\section{References}

Bretagnon, P. (1982) Astron. Astrophys., Vol. no. 114, p. 278-288.

Bretagnon, P., Francou, G. (1987) Astron. Astrophys., Vol. no. 202, p. 309-315.

McCarthy, D.D. (1992), IERS Standards (1992) IERS Technical Note . 13, Observatoire de Paris

Simon, J.L. (1983) Astron. Astrophys., Vol. no. 120, p. 197-202.

Simon, J.L., Bretagnon, P. (1984) Astron. Astrophys., Vol. no. 138, p. 169-178.

Simon, J.L., Joutel, F. (1988) Astron. Astrophys., Vol. no. 205, p. 328-334.

Simon, J.L., Joutel, F., Bretagnon, P. (1992) Astron. Astrophys., Vol. no. 265, p. 308323.

Standish, E.M. (1982) Astron. Astrophys., Vol. no. 114, p. 297-302.

Standish, E.M. (1994), Private Communication 